\title{
The SOA-Based Knowledge Management in the VROs
}

\author{
Li Wang, Jiaguyue $\mathrm{Xu}$, Junzhi Li and Guoping Xia \\ School of Economic and Management, Beihang University, Beijing 100083, P.R. China \\ wlbh@vip.163.com xjgy118@gmail.com leo.liz@hotmail.com gxia@buaa.edu.cn
}

\begin{abstract}
We analyze the features of knowledge management in the Virtual R\&D Organizations (VROs) and the features about the Service Oriented Architecture (SOA) in this paper. The application of SOA in knowledge management in VROs is further discussed. We process the registration, matching, and the construction of the UDDI in the knowledge service of VROs with SOA. Finally, we present a framework for the VROs to develop the knowledge management system with SOA.
\end{abstract}

Keywords: Knowledge management, Virtual $R \& D$ organizations, Serviceoriented architecture, Component-based service

\section{INTRODUCTION}

The Virtual R\&D Organizations (VROs) facilitates the resource sharing between various organizational subunits in the whole organizations with the advanced technology in cooperation and collaboration. The knowledge management in the VROs plays an important role in improving the core competitive advantage in companies. With the knowledge management system, the data, information and knowledge existed internally in single technique workers and experts transmit into a shared knowledge asset. The service aimed at resource cooperation and collaboration can support the technology innovation in the VROs efficiently [1].

The way to model the process of knowledge management, manage the distributed and related data dynamically is the key problem involved in the construction of the knowledge management system in VROs. The majority researches on the VROs primarily focus on the system structure, the concept and the description of the VROs. Samir Tata provides three knowledge management models integrated data, processing and knowledge [2]. These models have mechanisms to facilitate the communication and cooperation in companies. Boughzala builds MeDICIS model to describe the data communication between companies [3]. Y.Kim, Harald and Rikard study on the knowledge sharing between the virtual companies and provide a conception framework [4]. We draw on the topic of the application about the VROs which is not so widely discussed.

The Service-Oriented Architecture (SOA) is groupware-based model to construct the distributed system. It is well accepted by the industries and academies for its merits in flexibility, reusability and extendibility [5]. The service in SOA is composed by a group of interactive services. Each service can be mapped into one or multi embodied operation functions. The services help companies reduce their maintain cost

Please use the following format when citing this chapter:

Wang, L., Xu, J., Li, J., Xia, G., 2007, in IFIP International Federation for Information Processing, Volume 255, Research and Practical Issues of Enterprise Information Systems II Volume 2, eds. L. Xu, Tjoa A., Chaudhry S. (Boston: Springer), pp. 1019-1028. 
without the overlap process and improve the reusability and sharing of the applications. Compared with the traditional distribute system, the SOA cooperative application would be more dynamic and opening. How to integrate the service to meet the complex and dynamic application requirement and to improve the quality of the assemble services is widely discussed in the research of service-oriented cooperative application $[6,7]$.

The composite service in SOA is aimed to integrate each independent service according to its semanteme and the relationship in logic into a high level aggregation of function [8]. There are some academic research works about this topic. The BPELWS4 [9] provides an Eclipse plugin to facilitate the users to compose the services. The users need to know some basic knowledge about programming in this way. Shankar [10] uses the rules-based services to describe the automatic composite service. The service in the method should be pre-described and does not own the capability automatically. However, the mentioned two methods are both just dynamical composition of the main service construction. They had some difficulties in modulate service dynamically which is very important in the knowledge management process. First, the developers of the service come from different fields, so they have different understanding and description about the services. Given that, the transparent using of the main service would be not so easy. Second, the lacking of the convenient and quick-responding matching method can not meet the users' requirements. When the service requirements can not be exactly met, the main service can not give a sub-service to the users. Third, the mechanism lacks the flexibility to correspond and manage dynamically the service, which enables it hard to keep path with the dynamic operation development and evolvement.

The SOA structure is an efficient way to solve the distribution knowledge management system problem in the VROs. In order to unite the SOA and the knowledge management system in VROs, there are lots of detailed problems which need a certain time research.

\section{THE FEATURE OF SOA AND SOA IN THE KNOWLEDGE MANAGEMENT}

\subsection{The Improvement of Composite Capability Based on SOA in the Knowledge Management}

The composing mechanism in SOA helps the combination of knowledge in different fields and in different levels. For the knowledge in VROs covers multi knowledge fields, people need to look up different related knowledge in the whole process. The mechanism enables the query about the knowledge easier than before.

The second generation of the WEB service framework includes criterions ---the WS-Reliabe Messing, WE-Policy, WS-Addressing, WS-Coordination, WS-Atomic Transaction, WS-Business Activity and WS-BPEL. Each criterion includes an extending module with one or multi features. The flexible combination allows 
building the solution to meet the real requirement quickly. The knowledge management system is a complex and evolving system. With the features in SOA, the structure, application, service and massage can be developed and optimized gradually. The gradual process lowers down the cost and risk to organizations.

SOA structure makes the operation flow flexibly automatic and adaptable. The service in knowledge management would play a self-existent logical union. Given that, the knowledge search, filtrate, combination, exhibition in the process can be divided into a serial of services, in which each service charges a part of the executive process.

\subsection{The Internal Reusability and Extendibility in the SOA}

The SOA supports the reusing of service. Meanwhile the composite service itself could be reused by higher level composition. SOA is supposed to create service which is less related with the operation process and the automatic solution. The independence of the services supports the reusability.

Extendibility is another important feature of the SOA. The SOA advocates adding service and fuse of other service-oriented applications to extend the solution. The knowledge management system updated in this satiation can be much easier. The loose coupling in SOA encourages the least dependence among all the services, so the logical extendedness of the service would be finished in smallest influence.

\section{THE KNOWLEDGE MANAGEMENT IN VROS}

\subsection{The Knowledge Management in the R\&D Assignment in VROs}

The knowledge management system of VROs is used to support the knowledge management in the R\&D assignments, especially, the knowledge query in some special ones. With the certain R\&D object, the knowledge management system facilitates the organizer of the assignment to find the related workers and knowledge in the VROs and to build different levels knowledge management systems for the team.

The whole process includes:

1. Describe the knowledge required in the assignment. It should base on the existed description of the various organizational subunits or the intelligent agents knowledge capabilities. As to the assignment, the description should be mined deeply and re-organized.

2. Construct the intelligent agents according to the knowledge and capabilities of the subunits members. The members in the intelligent agents may come from one various organizational subunits, either from different subunits.

3. Disassemble the whole assignment according to the capability of the intelligent agents. Each sub-assignment should have an intelligent agent to finish. The disassemble assignments should be saved in a mixed list. 
4. Build the subjective task view with the sub-assignments construct information.

5. Build or rectify the knowledge management subsystem to each intelligent agent.

6. Build the relationship between the knowledge management subsystem and the whole VROs knowledge management system and the cooperation mechanism.

7. Build the sharing mechanism and environment for the members to communicate the tacit knowledge.

8. Collect the new born knowledge in the R\&D process. Then, assess the knowledge, and list the knowledge which is essential to finish the assignment.

\subsection{THE KNOWLEDGE MANAGEMENT FRAMEWORK IN VROS}

\subsubsection{The SOA Based Knowledge Management System}

SOA is an efficient way to manage the knowledge in VROs. The SOA allows the system to build a lots of atom service, and provide composite service in different levels of knowledge management with these atom services. The composite service could be in all levels of the internal knowledge management systems, also between the internal organization and intelligent agent knowledge management systems.

The division of the R\&D assignments and the matching between the assignments and the organizational subunits either intelligent agent is the main problem in the reconstruction of the knowledge resource in VROs. In order to utilize the resource of the members in different organizational subunits and intelligent agents in different locations, each assignment should be defined clearly according to the features of the assignments and the capability of the organizational subunits and intelligent agents. Meanwhile, the whole R\&D process should be divided into several sub-assignments which are independent to each other. The loosing coupling feature of SOA enables the assignment decomposition to become efficient to build the losing coupling relationship between the sub-assignments and the service composition in the knowledge management system.

\subsubsection{The Registration, Querying and Matching of the Service}

The SOA includes service composition, orchestration, and choreography, which are used to build the relationship between the sub knowledge management systems and the whole organization knowledge management system. Usually, each organizational subunit either the intelligent agent does not only copper with knowledge management affairs internally, but also deal with the entire knowledge management from the cooperation mechanisms and protocols.

The matching between the knowledge requirement and the capability of the organizational subunit either the intelligent agent is actually the selection in capability and resource. In this case, it is important that the way in which the system could find the right organizational subunits and the intelligent agents. If we take the qualitative search arithmetic, the insufficiency of the knowledge description in using the keywords matching way reduces the relativity between the search result and the best results. The Universal Description, Discovery, and Integration (UDDI) API can solve this problem and provide more search mechanisms. 
The organization need to build the UDDI public knowledge service registration center in VROs. There are also private UDDI centers in each organizational subunit and intelligent agents. The private UDDI center can only be used locally or authorizedly.

\subsubsection{The Knowledge Service Registration Center UDDI}

UDDI supports new sort pattern and mark pattern. Because the members in different organizational subunits and the intelligent agents in the VROs are skilled at different specialties, the knowledge management in VROs involves different ways of category and marking. According to the features in VROs, the knowledge management in the organizational subunits and intelligent agents is designed to separate relatively. Meanwhile, the UDDI should meet the knowledge sharing requirement in the VROs.

First, the members in organizational subunits or intelligent agents register the technology information about some classifications into the public UDDI, which the members of organizational subunits or intelligent agents have already make an agreement on. And the knowledge management system should apply the taxonomy validation service in the UDDI. Second, each member in organizational subunits and intelligent agent registers their own information in to UDDI and the system uses the same way as first step to classify the information. Third, The UDDI verifies the information and classification.

Among the process, the classification in the knowledge management describes the knowledge in diversified dimensionalities. Tacit knowledge is related with the scale of the knowledge including the depth and span, the point of view, the particularity degree including the precision and granularity. Explicit knowledge is described in the facets of application context, case and so on.

The process of the $R \& D$ assignment required knowledge, the members in organizational subunits and intelligent agents matching is formulated as following:

Definition 1: The cooperation R\&D assignment (T): The assignment the VROs required its members to finish it in a certain time. $T$ is a limited set.

$$
\mathrm{T}=\left\{\mathrm{T}_{1}, \mathrm{~T}_{2}, \ldots, \mathrm{T}_{\mathrm{i}}\right\}, \mathrm{i} \geq \mathbf{1}
$$

$i$ presents the ith cooperation $R \& D$ assignment

Definition 2: The knowledge demand in the R\&D assignment: The knowledge is required by the $R \& D$ assignments. $D$ is a set:

$$
\mathrm{D}(\mathrm{T})=\left\{\mathrm{D}\left(\mathrm{T}_{1}\right), \mathrm{D}\left(\mathrm{T}_{2}\right), \ldots, \mathrm{D}\left(\mathrm{T}_{\mathrm{i}}\right)\right\}
$$

$D\left(T_{i}\right)$ present the knowledge required in ith assignment. The $D\left(T_{i}\right)$ should be fractionized into smaller set.

$$
D\left(T_{i}\right)=\left\{d\left(T_{i}\right) 1, d\left(T_{i}\right) 2, \ldots, d\left(T_{i}\right) j\right\}
$$

$d\left(T_{i}\right) j$ presents the smaller knowledge requirement.

Definition 3: The capability in members in organizational subunits or intelligent agent $(\mathrm{C})$ :

$$
\mathrm{C}(\mathrm{O})=\left\{\mathrm{C}\left(\mathrm{O}_{1}\right), \mathrm{C}\left(\mathrm{O}_{2}\right), \ldots, \mathrm{C}\left(\mathrm{O}_{\mathrm{j}}\right)\right)
$$

$\mathrm{C}\left(\mathrm{O}_{\mathrm{i}}\right)$ present the its members or intelligent agent's capability. The $\mathrm{C}\left(\mathrm{O}_{i}\right)$ should be fractionized into smaller set.

$\mathrm{c}\left(\mathrm{O}_{\mathrm{i}}\right) \mathrm{k}$ presents the smaller capability cell.

$$
\mathrm{C}\left(\mathrm{O}_{\mathrm{i}}\right)=\left\{\mathrm{c}\left(\mathrm{O}_{\mathrm{i}}\right) 1, \mathrm{c}\left(\mathrm{O}_{\mathrm{i}}\right) 2, \ldots, \mathrm{c}\left(\mathrm{O}_{\mathrm{i}}\right) \mathrm{j}\right\}
$$


With the definitions, the matching between the knowledge requirement and the capability in organizational subunits and the intelligent agent can be described as the mapping relation:

$$
\mathrm{D}\left(\mathrm{T}_{\mathrm{i}}\right) \Leftrightarrow \mathrm{C}\left(\mathrm{O}_{\mathrm{i}}\right)
$$

In order to enable the total amount of the capability to cover the whole knowledge requirements, the $C(O)$ should be registered in the UDDI center. According to $D(T)$, the search engine uses the semantic and ontology search arithmetic to find and build the mapping relation.

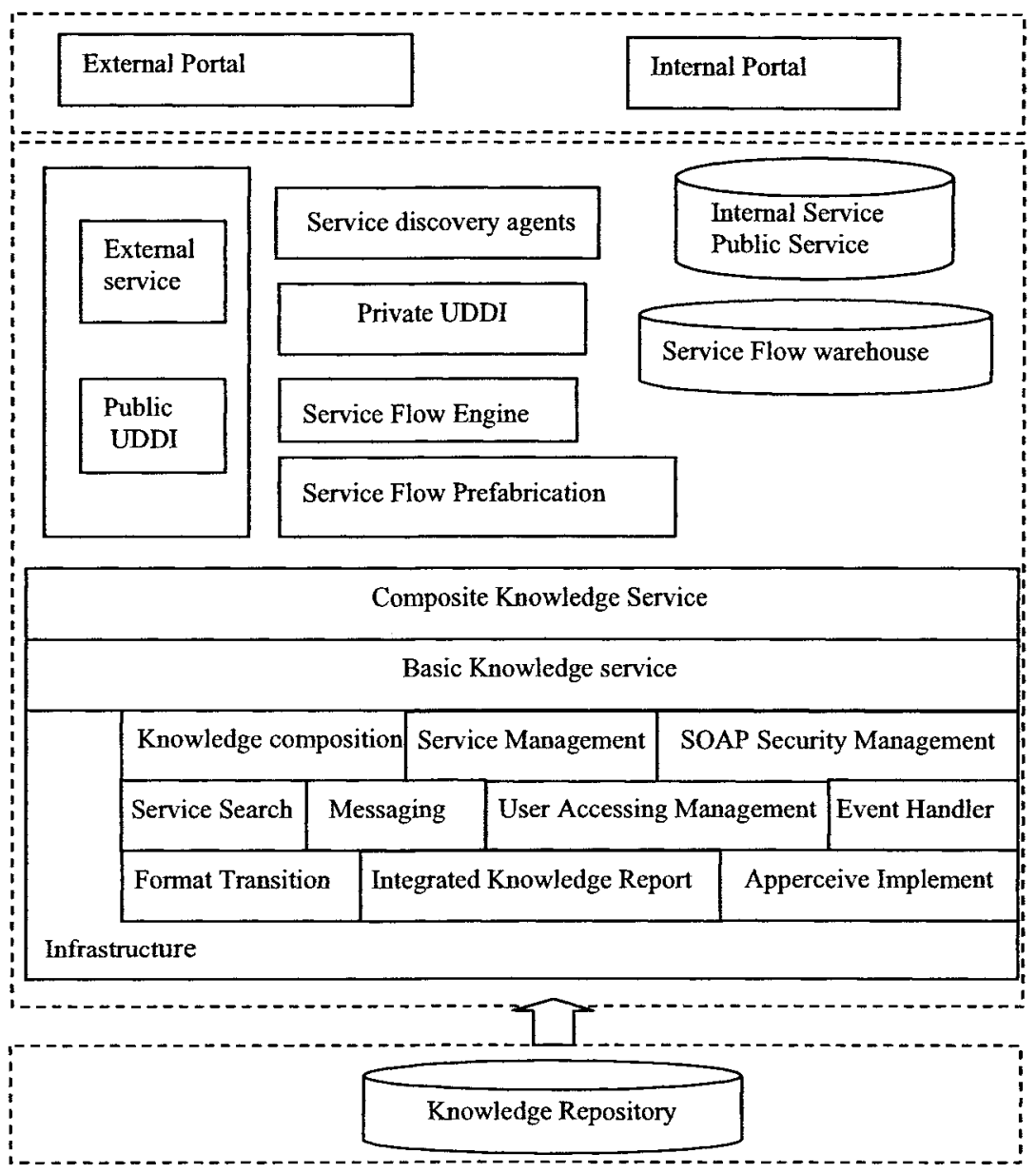

Figure 1. The Framework of Knowledge Management in VROs 
Figure 1 shows the framework of the VROs. The process of the knowledge service includes the Atom Service (AS), the infrastructure and the knowledge repository. The level of atom service can provide knowledge service publicly. There is some knowledge queries which can not be answered by single AS. The service flow engine composites the service according to such kinds of user queries. The composite service has two kinds---the prefab and the programmed. The prefab service is the composite service which designed in the development. The programmed service is the one which the clients use the service flow engine to compose the service. The process is controlled by the service flow engine. The infrastructure includes all the components to conduct the knowledge service and support the calling from the up level components.

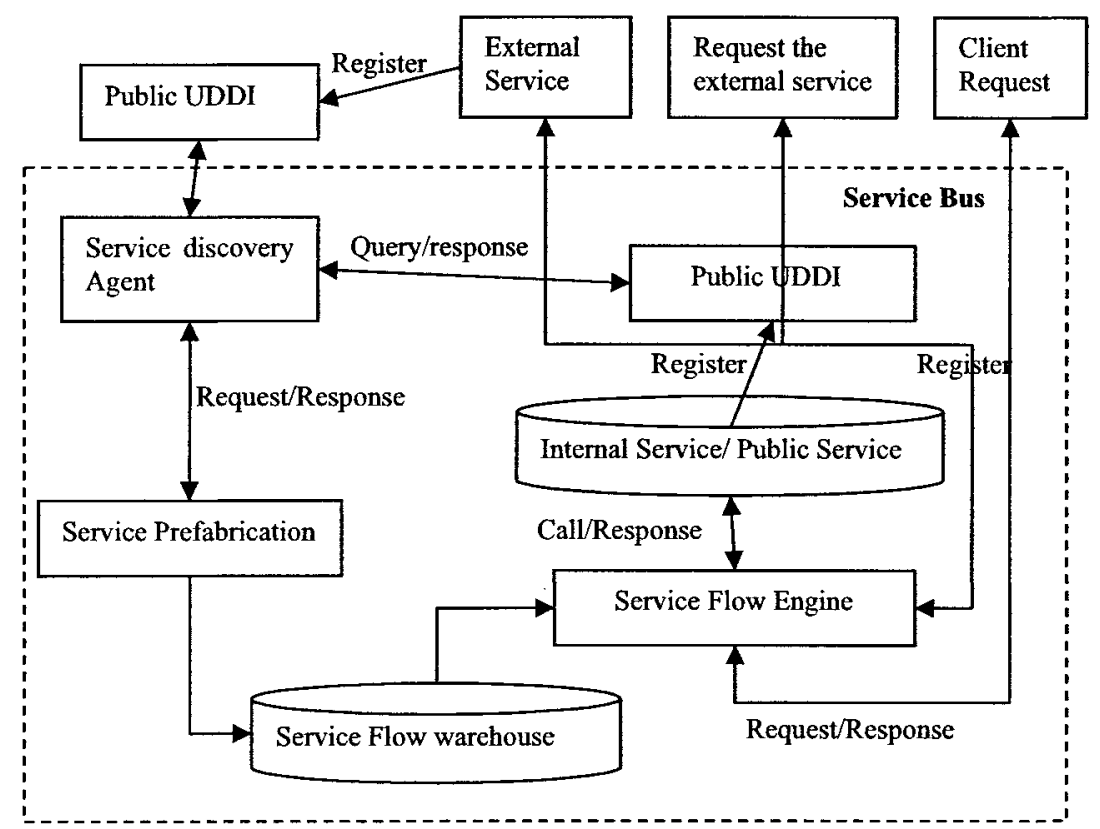

Figure 2. The Service Bus Framework

Figure 2 illustrate the work of the service bus. The service bus refers to a software architecture construct, implemented by application of the local operation process to service the users. There are one private UDDI and a service found agent in the service bus. The private UDDI is used to register internal knowledge services in the organizational subunits and the intelligent agent as well as the local public service. The service discovery agent finds the knowledge first in the private UDDI. If it could not find, the agent would turn to the external UDDI. Sometimes, there would be more than one services meeting the requirement. In case that, the service discovery agents need to range the service in the service agreements. The local public service should be registered in the public UDDI for the public using. 
The service flow prefab machine finds the relevant service according to the operation process logic, in the processing service which needs to deal with previously.

In both the external and local web applications, the service flow engine is started after it goes into the service bus. The service flow engine firstly charges the kinds of the service---the prefab, the basic or the program-required. If it is the prefab, the engine would execute the existed service flow; if it is the basic, it should run directly; if it is the program-required, the engine needs to search and select.

\section{THE APPLICATION OF THE KNOWLEDGE MANAGEMENT IN VROS}

The Three-gorge Dam in China is one of the biggest hydroelectricity engineering in the world. There is growing recognition that the hydroelectricity engineering is a knowledge-intensive process that requires the integration of specialized knowledge in R\&D. As the main undertaker to the project, Chinese Gezhouba Group Cooperation(CGGC) needs to use the SOA based VROs knowledge management system to solve problems in efficient ways. The knowledge management system can register the sub-organizations knowledge capability and provide the query function to search the required capability to finish the R\&D assignments. Also, the knowledge management system can build intelligent agents---the new project departments--to work on the assignment. Using the composite service technology in SOA to combine the sub-organization with the existed single knowledge service, and change it into an operation logical based complex composite service to work on the technology problems.

Figure 3 illustrates the construction of the knowledge repository subsystem in the SOA based VROs knowledge management system in CGGC. The knowledge repository has the function to store, modify, delete and deduce knowledge, based on the reasonable presentation and efficient management about the knowledge in the hydroelectricity engineering. The knowledge sharing in the VROs would be from encapsulating the knowledge deduction function into a service and releasing the service. The knowledge repository is a kind of application of the Artificial Intelligence $(\mathrm{AI})$ in the database field. We can consider it as an intelligent database. The knowledge repository involves two parts---one extension database and intension database. The process of modeling the knowledge repository is special from the normal database. The extension database stores the data in traditional database, which we call it storing the facts and the relationship in such database real relationship. The intension database does not store date, but the rules, from which we can get new date. The data in the intension database would be available after the deduction. That is why we call it virtual relationship [12]. The knowledge repository and the transition database are both in the same database management system. There are two ways for knowledge acquisition. The knowledge can be automatically extracted from the transition database or external database. Also, the knowledge engineering could input the knowledge into the system directly. The knowledge management in CGGC is based on the J2EE platform with the adding, deleting, modifying and querying 
function. Actually, ete result of the knowledge querying is the result of the knowledge management system's virtual data. The knowledge is encapsulated into the Web service and the service is registered in the UDDI registration center. Then the service becomes the knowledge service which could be released to the public. The security and massage management would be achieved by the service management software.

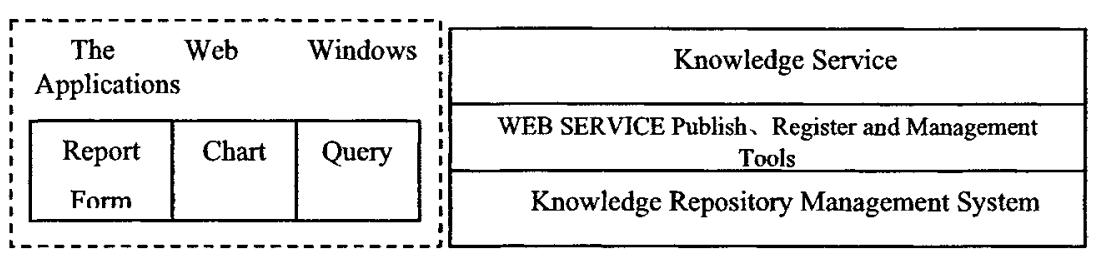

WEB LOGIC J2EE Platform

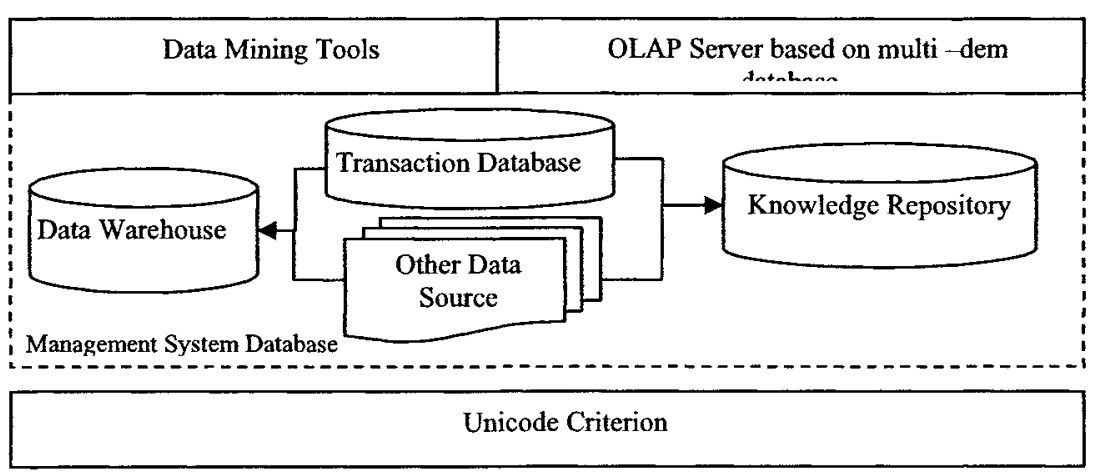

Figure 3. The Knowledge Repository Management System

\section{CONCLUSIONS}

The knowledge management system in the VROs based on the SOA can improve the level of knowledge sharing in the VROs. From the sharing platform, the cost in development can reduce with the composite service dynamically. The flexibility makes the VROs to keep path with the changing environment more easily. The research in the knowledge management system with SOA can lead the development of the VROs management to be more cooperative and service-oriented. 


\section{REFERENCES}

1. H. Smyth and R. Longbottom, External Provision of Knowledge Management Information Services: The Case of the Concrete and Cement Industries, European Management Journal. Volume 23, Number 2, pp.247-259, (2005).

2. S. Tata and I. Boughzala, Modeling Contribution for Virtual Enterprise Support, in Proc. of the Twelfth IEEE International Workshops on Enabling Technologies: Infrastructure for Collaborative Enterprises, eds. R. Werner(IEEE: New York, 2003), pp.165-170.

3. I. Boughzala, A Methodology for Designing Interagent Cooperative Information System for Knowledge Management. Ph.D thesis, Pierre et Marie Curie Paris VI University (2001).

4. Y. Kim, H. Lee, and I. Han, Virtual and Knowledge-Based Organization Minitrack, in Proc. of the 33rd Hawaii International Conference on System Sciences, eds. R.H. Spraque (IEEE: New York, 2000), p.6060.

5. M.P. Papazoglou and D. Georgakopoulos, Service oriented computing, Communications of the $A C M$ (S0001-0782).Volume 46, Number 10, pp.25-28, (2003).

6. G. Chafle, S. Chandra, and V. Mann, Decentralized orchestration of composite Web Services, in Proc. of the 13th International World Wide Web Conference, eds. S.I. Feldman, M. Uretsky, M. Najork, and C.E. Wills(ACM: New York, 2004), pp.134-143.

7. H. Zhu, Challenges to Reusable Services, in Proc. of 2005 IEEE International Conference on Services Computing (IEEE: New York, 2003), p.120.

8. F. Casati, S. Ilnicki, L. Jin, V. Krishnamoorthy, and M. Shan, Adaptive and dynamic service composition in $\mathrm{eFlow}$, in Proceedings of the International Conference on Advanced Information Systems Engineering, eds. B. Wangler, L. Bergman(Springer: Boston, MA, 2006), pp.13-31.

9. T. Andrews, G. Cubera, and H. Dholakia, Business process execution language for Web services, Version 1.I. BPEL4WS specification, IBM (2005).

http:/www.ibm.com/developerworks/library/ws-bpel/ (Accessed July 15, 2007).

10. R.P. Shankar and F. Armando, SWORD: A developer toolkit for web service composition, in Proc. of the 11th International World Wide Web Conference (ACM: New York, 2002), pp.83-107.

11. E. Newcomer and G. Lomow, Understanding SOA with Web Services (Pearson Education Inc: Old Tappan, NJ, 2005).

12. Anonymous, OASIS functional elements specification (committee draft2.0), OASIS(2006). http:/www.oasis-open.org/apps/org/workgroup/fwsi/documents.php (Accessed July 15, 2007) 